\title{
On the integrability of halo dipoles in gravity
}

\author{
Werner M. Vieira* and Patricio S. Letelier ${ }^{\dagger}$ \\ Departamento de Matemática Aplicada \\ Instituto de Matemática, Estatística e Ciências da Computação \\ Universidade Estadual de Campinas, CP 6065 \\ 13081-970 Campinas, SP, Brazil
}

September 17, 1996

\begin{abstract}
We stress that halo dipole components are nontrivial in core-halo systems in both Newton's gravity and General Relativity. To this end, we extend a recent exact relativistic model to include also a halo dipole component. Next, we consider orbits evolving in the inner vacuum between a monopolar core and a pure halo dipole and find that, while the Newtonian dynamics is integrable, its relativistic counterpart is chaotic. This shows that chaoticity due only to halo dipoles is an intrinsic relativistic gravitational effect.
\end{abstract}

PACS numbers: 04.20.Jb, 05.45.+b, 95.10.Fh, 95.30.Sf

Typeset using REVTEX

*e-mail: vieira@ime.unicamp.br

†e-mail: letelier@ime.unicamp.br 
We report in this Letter a simple situation where the contact between Newton's gravity and General Relativity (GR) allows for a clear distinction between their predictions about chaotic behavior of orbit dynamics.

We considered in [1] how does GR deal with a monopolar core (at first approximation), which may be a neutron star, a galactic nucleus or even a black hole, surrounded by a distant, massive halo of dust. We exhibited an exact, static, axially symmetric model with a halo made of quadrupoles and octopoles of arbitrary strengths. In constructing this solution, we were also guided by its formal resemblance to the classical Hénon-Heiles potential in the Newtonian limit.

Here, we are mainly interested in the dynamical rather than formal comparison between Newtonian core-halo systems and its relativistic counterpart. Firstly, we state the problem according to Newton's theory: let the coordinate origin stay at the mass center of the monopolar core, $z$ be the symmetry axis of the core-halo system and $D$ be the region between the minimum and the maximum spheres centered at the origin that isolate the inner vacuum from the core and the halo. We have to solve Laplace's equation in $D$ for the axially symmetric Newtonian potential. By using standard spherical multipolar expansion, we arrive at the following gravitational potential felt by test particles evolving in $D$ :

$$
\Phi_{N}=\Phi_{0}-\frac{1}{\rho}-\mathcal{D} z+\frac{\mathcal{Q}}{2}\left(2 z^{2}-r^{2}\right)+\frac{\mathcal{O}}{2}\left(2 z^{3}-3 z r^{2}\right)+\cdots .
$$

$\Phi_{0}$ is a constant (put here only for the sake of completeness) and $\rho^{2}=r^{2}+z^{2}=x^{2}+y^{2}+z^{2}$ where $x, y, z$ are the usual Cartesian coordinates. $\Phi_{N}$ and all quantities in it are written in convenient nondimensional units. $\mathcal{D}, \mathcal{Q}$ and $\mathcal{O}$ are respectively the dipole, quadrupole and octopole halo strengths given by the appropriate normalized integrals over the halo (as a source) in the Newtonian approach. It is worth to stress that, quite apart from the nomenclature, the halo multipoles have opposite behavior in $D$ from the usual decreasingwith-distance core multipoles. In particular, by merely translating the origin to the core mass center we can (and in fact we do) eliminate the usual core dipole in $D$, so that the next higher core contribution should be the usual quadrupolar one (which will not be considered 
here). On the other hand, nothing so simple can eliminate the halo dipole component. As it is easily seen, the permanency of the later is related to the halo mass distribution with respect to the plane $z=0$. The only way of eliminating halo dipoles from (1) should be passing to a noninertial reference frame with uniform acceleration $\mathcal{D}$ in the $z$-direction. In this case, we should have to properly treat this problem as a relativistic one, since it extrapolates the Galilean gauge invariance of the Newtonian physics.

Now, we consider bounded orbits under the Newtonian potential (11). If we switch the halo quadrupole and octopole off $(\mathcal{Q}=\mathcal{O}=0)$, thus the remaining potential describing the core plus the pure halo dipole is integrable, in particular being separable in parabolic coordinates [2]. The hamiltonian $H$ as well as the additional constant of motion $C$ in involution with the hamiltonian are

$$
\begin{array}{r}
H=\frac{1}{2}\left(p_{r}^{2}+p_{z}^{2}\right)+\frac{\ell^{2}}{2 r^{2}}+\Phi_{N}, \\
C=r p_{r} p_{z}-z p_{r}^{2}-\ell^{2} \frac{z}{r^{2}}+\frac{z}{\rho}-\frac{\mathcal{D}}{2} r^{2},
\end{array}
$$

where $\ell$ is the nondimensional conserved angular momentum associated to the axial symmetry. Only to illustrate the integrable dynamics, we present in Fig. 1(a) a typical $z=0$ Poincaré section for this case. Contrasting with this, we mention that either the Newtonian halo quadrupole $(\mathcal{Q} \neq 0)$ or octopole $(\mathcal{O} \neq 0)$ does produce chaos, which will be shown in an extended forthcoming paper.

Since the Newtonian halo dipole is nontrivial in $D$, appearing quite naturally in (1), we ask for its relativistic counterpart. In fact, we succeeded in extending our previous relativistic solution [1] to add a dipole term to the halo. Starting from Weyl's metric and following the formulation presented therein, the solution depends upon two functions $\nu(u, v)$ and $\gamma(u, v)$, the last being obtained by quadrature from the former one. Here, it is sufficient to write down only the main function $\nu(u, v)$ (see [1] for notation and details):

$$
\nu(u, v)=a_{0} Q_{0}(u)-\mathcal{D} P_{1}(u) P_{1}(v)+(2 / 3) \mathcal{Q} P_{2}(u) P_{2}(v)+(2 / 5) \mathcal{O} P_{3}(u) P_{3}(v) .
$$

This equation should be compared with eq. (3) of that Reference. Essentially, the first term 
with $a_{0}=-1$ describes the monopolar core (that can be switched off by putting $a_{0}=0$ ), the remaining ones being the corresponding multipoles originated from the halo. $Q_{0}$ and $P_{n}$ are the corresponding Legendre's functions in standard notation and $u, v$ are prolate spheroidal coordinates constructed from Weyl's ones.

What is the Newtonian limit of (3)? The basic steps to obtain it are: i) The relativistic solution is valid in the full vacuum between the core and the halo. ii) We assume that there exist a region $\bar{D}$ in the intermediate vacuum where the conditions of weak gravitational field and slow motion of test particles occur. Then, Eintein's equations reduce in $\bar{D}$ to Laplace's equation for the Newtonian potential $\Phi_{N}$, which relates to the metric through the temporal component $g_{t t}=1+\left(2 / c^{2}\right) \Phi_{N}$ (the remaining spatial components of the metric being irrelevant to this approximation). iii) The Schwarzschild coordinates at which we finally come back with the solution approximate to the usual Euclidean spherical ones plus time in $\bar{D}$. We consistently assume that this region is far from the core's event horizon. Now, by applying all this to the relativistic solution (3) and expanding $g_{t t}$ to the first order in $\mathcal{D}$, $\mathcal{Q}$ and $\mathcal{O}$ we arrive, after the conversion to nondimensional units, exactly at the potential $\Phi_{N}$ given by (1) (apart from the irrelevant constant $\Phi_{0}$ ). The implicit assumption here is that $\bar{D}$ and the Newtonian region $D$ above have a nonempty intersection at least. We would like to stress the nontrivial character of halo dipoles also in the relativistic context, as they contribute to the Kretschmann's scalar $R^{\alpha \beta \gamma \delta} R_{\alpha \beta \gamma \delta}$ even if $a_{0}=\mathcal{Q}=\mathcal{O}=0$. Moreover, they are smooth, however exact, relativistic counterparts of Newtonian halo dipoles, in the sense that their first contribution to the Riemann tensor is of second order in $\mathcal{D}$.

Next, we compare bounded geodesics generated by (3) for a pure halo dipole $(\mathcal{Q}=\mathcal{O}=0)$ with the preceding Newtonian case. A typical Poincaré section of relativistic orbits is shown in Fig. 1(b) where vast chaotic layers are seen in the geodesic motion. This should be contrasted with the full integrability of the corresponding Newtonian figure 1(a). Since Poincaré sections are global, gauge invariant tools to detect chaos in bounded systems, this proves that chaoticity in the vacuum between a monopolar core and a purely dipolar halo of dust is an intrinsic relativistic gravitational effect. 
An extended numerical study of both newtonian and relativistic cases as well as the details of the full relativistic solution (3) will be presented in an extended forthcoming paper.

The authors thank CNPq and FAPESP for financial support. 


\section{REFERENCES}

[1] Vieira, W. M. and Letelier, P. S., Phys. Rev. Lett. 76, 1409 (1996).

[2] Grammaticos, B., Dorizzi, B., Ramani, A. and Hietarinta, J., Phys. Lett. 109A, 81 (1985). 


\section{FIGURES}

FIG. 1. Poincaré sections of orbits in the vacuum between a monopolar core and a pure halo dipole $(\mathcal{Q}=\mathcal{O}=0)$ through the plane $z=0$ (for $p_{z}>0$ ): (a) the Newtonian case in Euclidean cilyndrical coordinates for the potential (11) with nondimensional orbit parameters $E=-0.03$ (energy), $\ell=3.8$ (angular momentum) and $\mathcal{D}=5 . \times 10^{-4}$ (halo dipole strength), and (b) the relativistic case for timelike geodesic motion in Weyl's coordinates for the solution (3) with $E=0.975$, $\ell=3.8$ and $\mathcal{D}=3 . \times 10^{-4}$. Note that the relativistic energy $E=0.975$ corresponds in the Newtonian limit to the energy $E=-0.025$. 


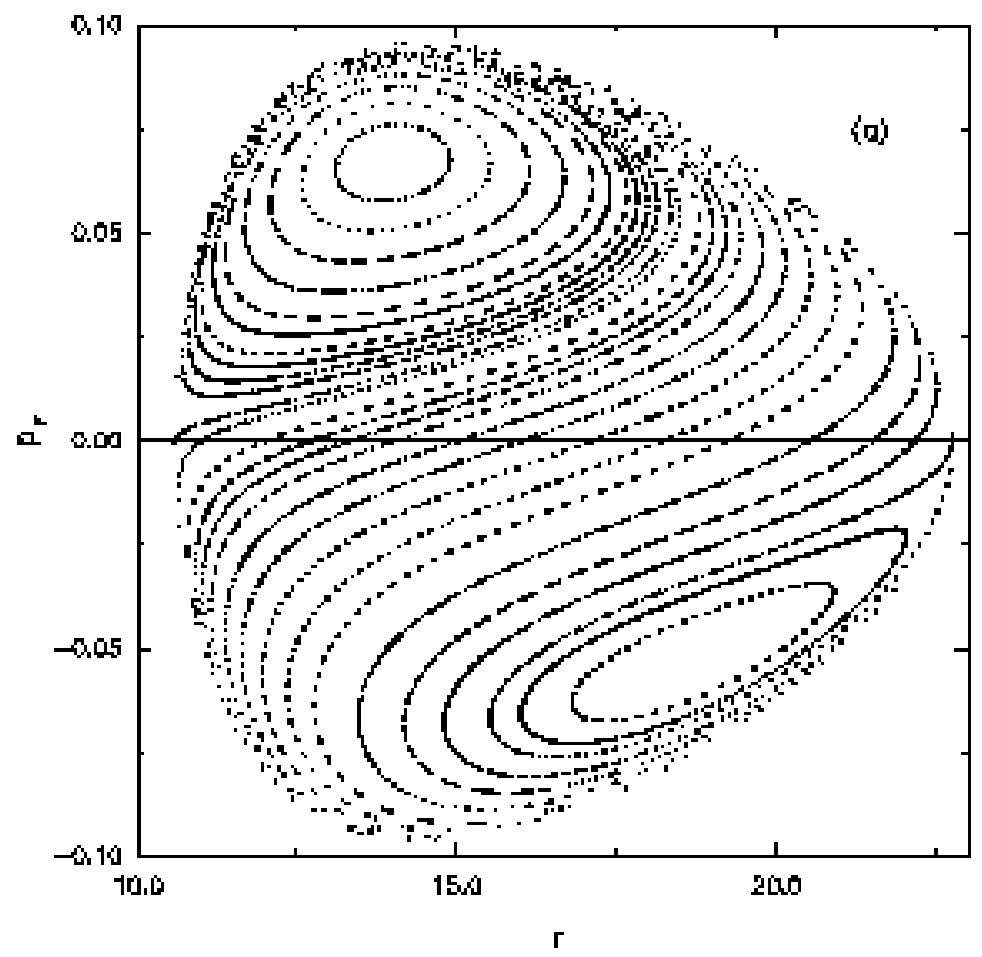




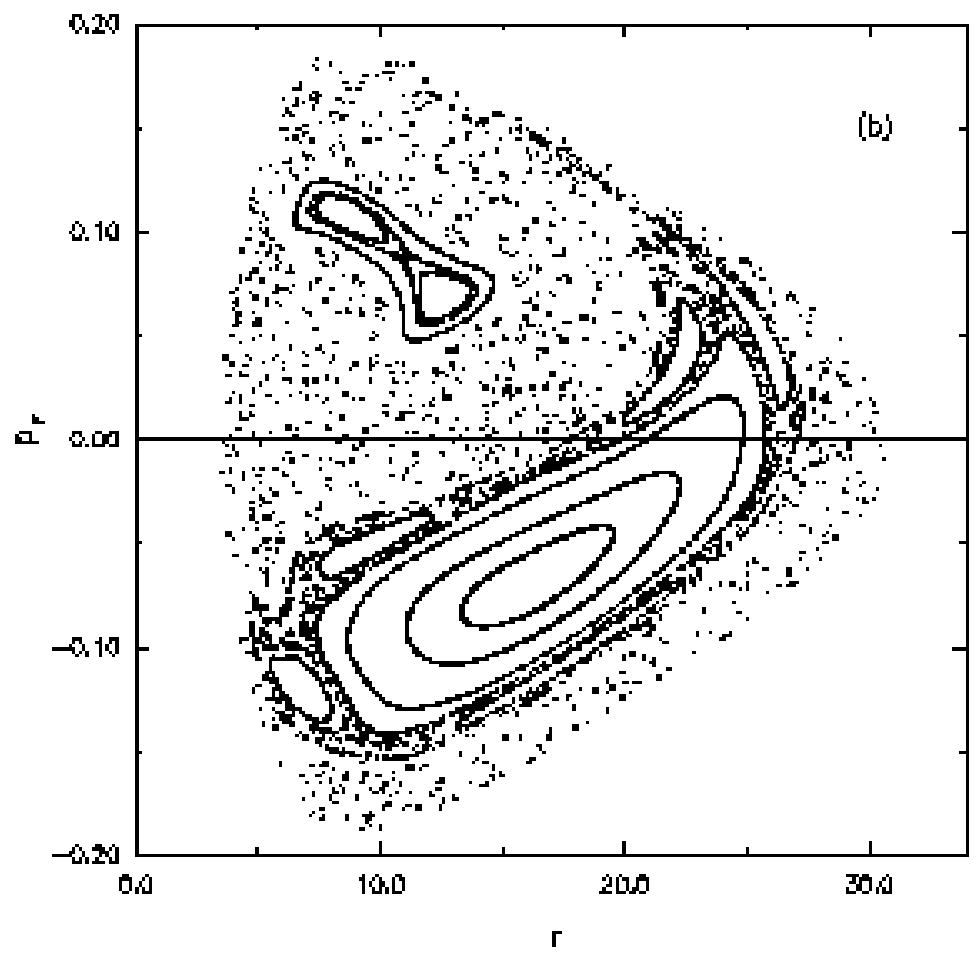

